\title{
Photons, dust, and honey bees
}

\author{
Diederik Wiersma
}

\author{
European Lab. For Non-linear Spectroscopy (LENS) \\ INO-CNR, Univ. of Florence
}




\section{Photons, dust, and honey bees}

\section{Diederik Wiersma}

European Lab. For Non-linear Spectroscopy (LENS) INO-CNR, Univ. of Florence www.complexphotonics.org 


\title{
Micro and nano photonics group
}

\author{
European Lab. For Non-linear Spectroscopy (LENS) \\ INO-CNR, Univ. of Florence \\ www.complexphotonics.org
}

Pierre Barthelemy

Matteo Burresi

Francesca Intonti

Rajesh Kumar

lacopo Malfanti

Vitantonio Matarazzo
Camilla Parmeggiani Francesco Riboli Romolo Savo Radha Vivekananthan Kevin Vynck

Jacopo Berlototti Silvia Vignolini
Stefano Cavalieri Marcello Colocci Massimo Gurioli Stefano Lepri Roberto Livi Roberto Righini 


\section{Optics of complex systems}

Light transport in dielectric materials

Disordered

$\downarrow$

Powders

Porous materials

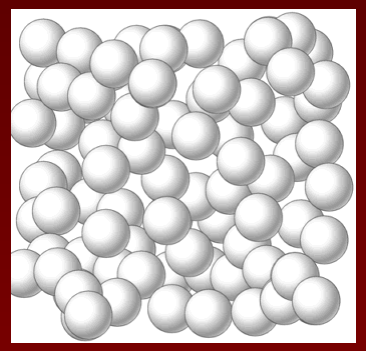

Partially ordered

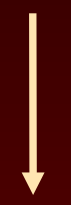

Liquid crystals

Quasi crystals

Ordered

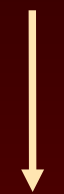

Photonic crystals
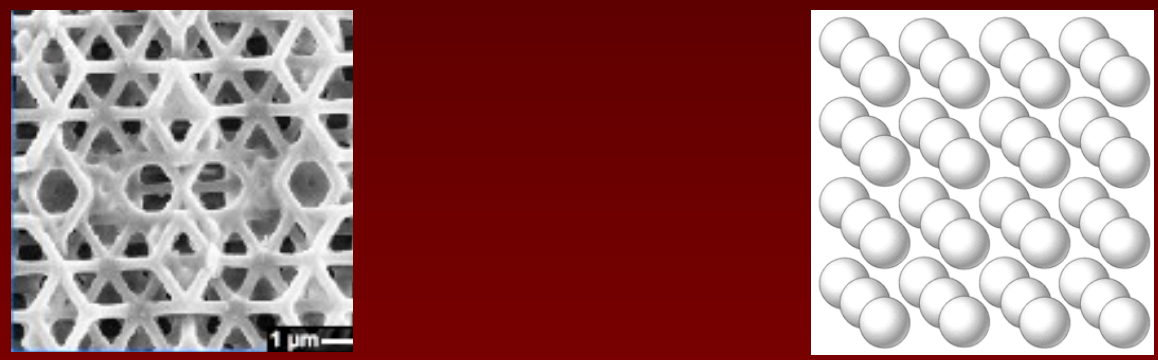


\section{Understanding light propagation}




\section{Understanding light propagation}

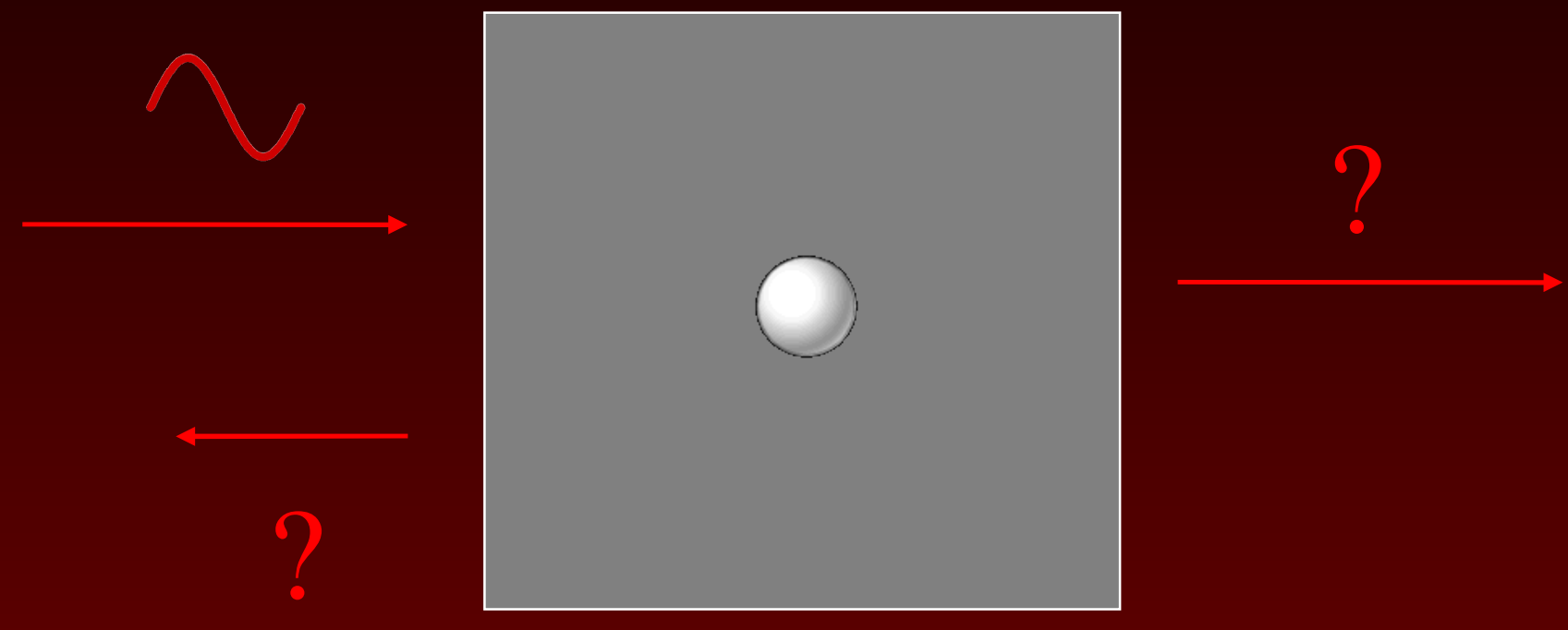

Single scattering 


\section{Understanding light propagation}
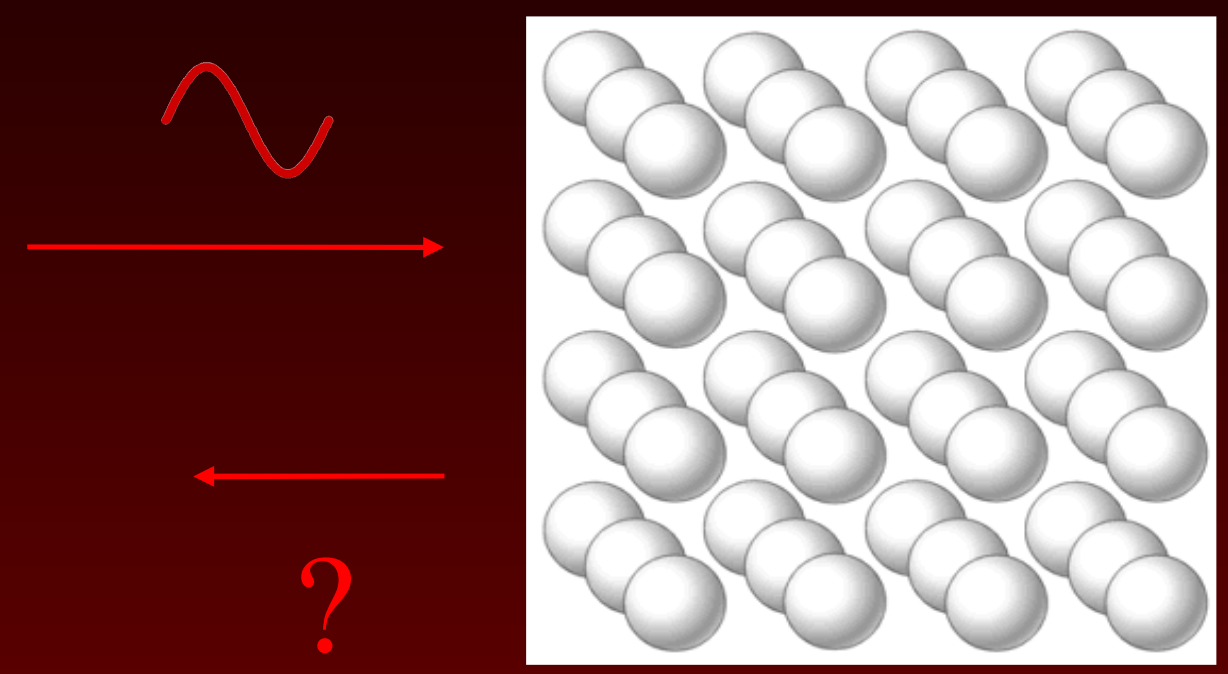

Photonic crystal 


\section{Understanding light propagation}

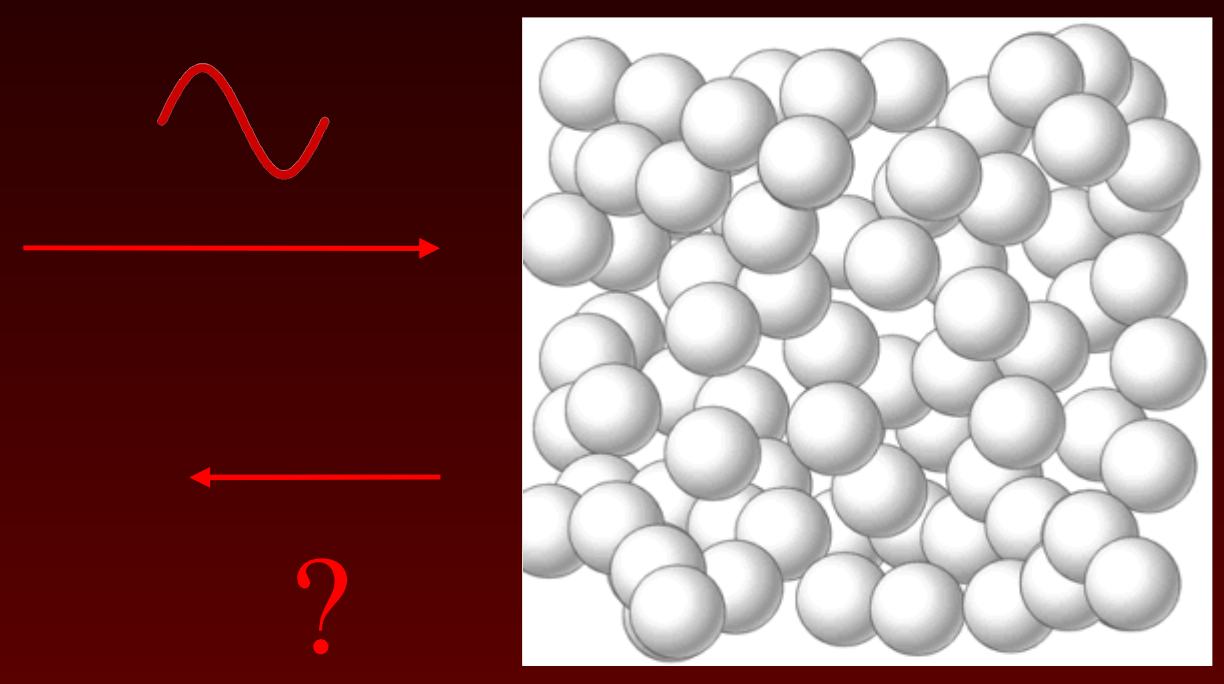

Photonic glass 


\section{Disordered materials}

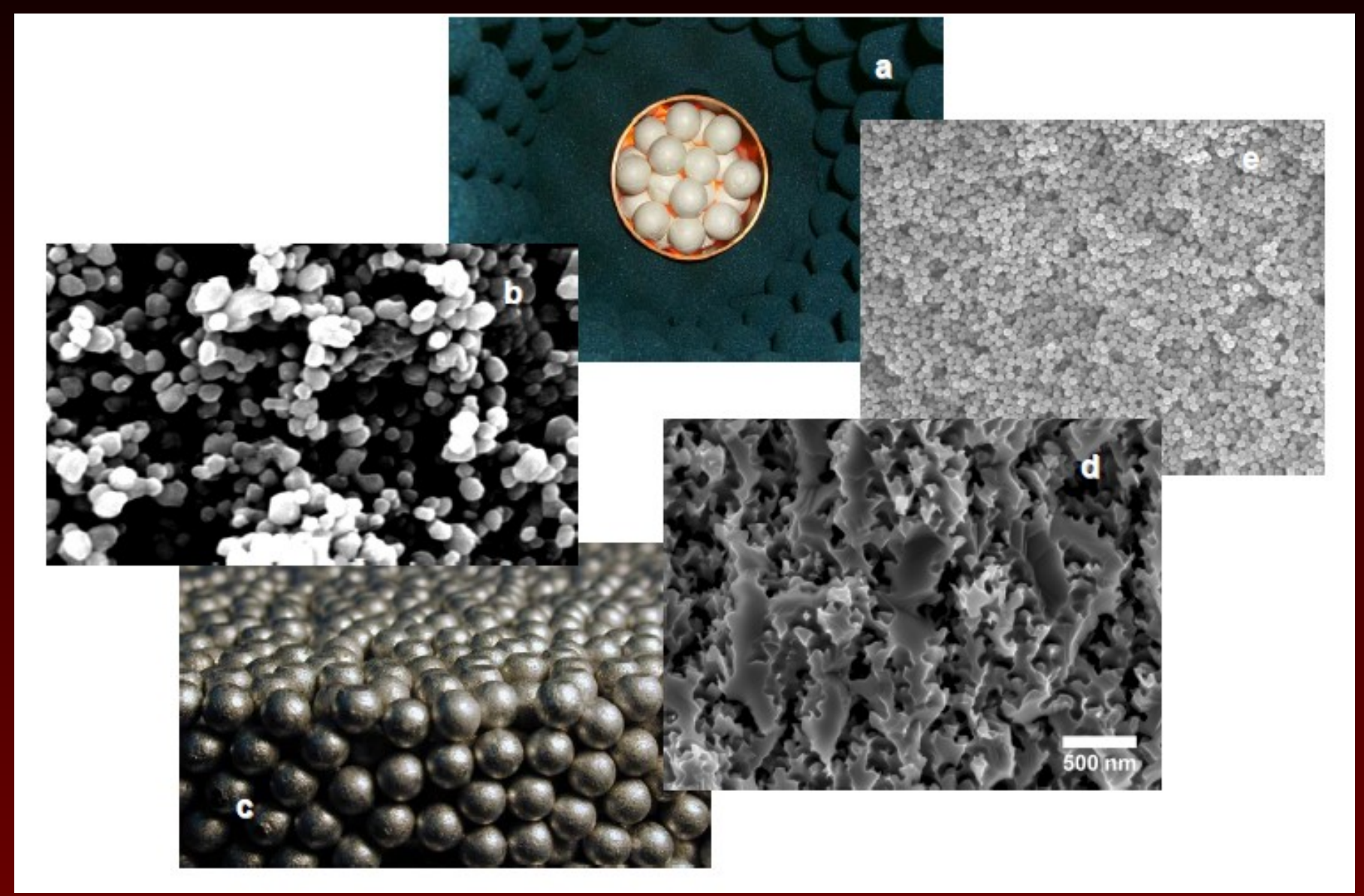

Physics Today, August issue (2009) 


\section{Random materials}

Light waves perform random walk

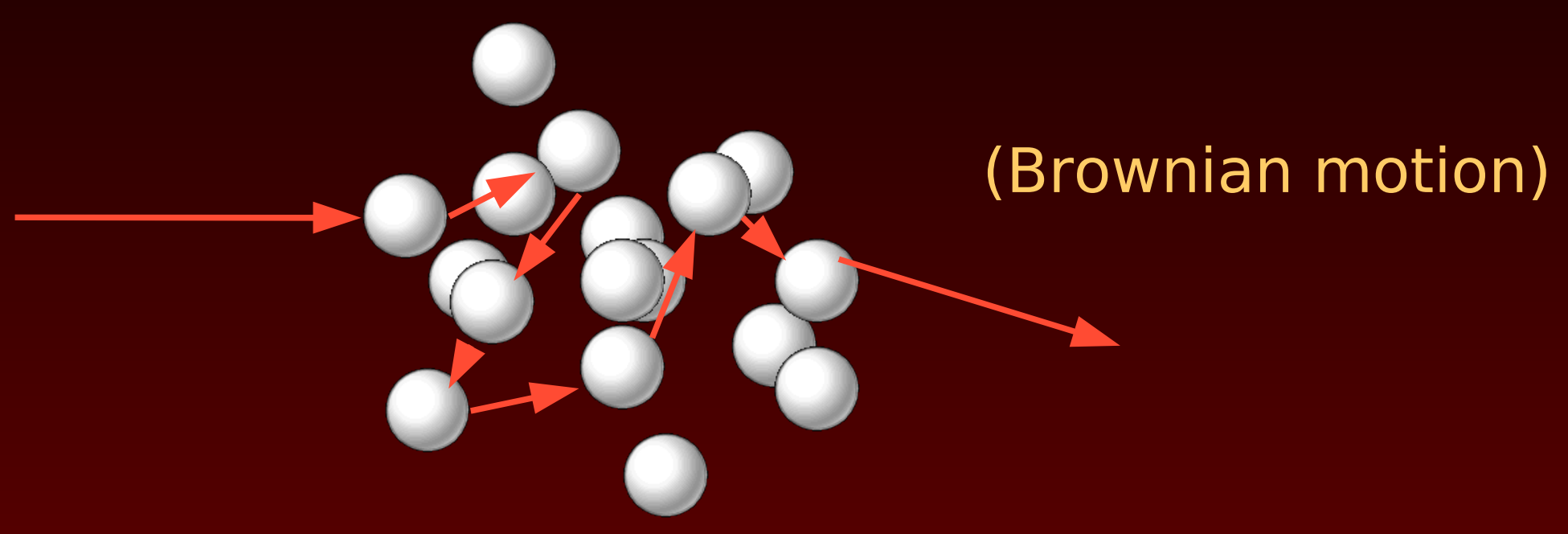

Average step $\ell$ (mean free path)

$\longrightarrow$ Diffusion process: $\left\langle x^{2}\right\rangle=D \cdot t^{\gamma} \quad$ with $D=\frac{1}{3} v \ell$ 


\section{Scattering}

single scattering

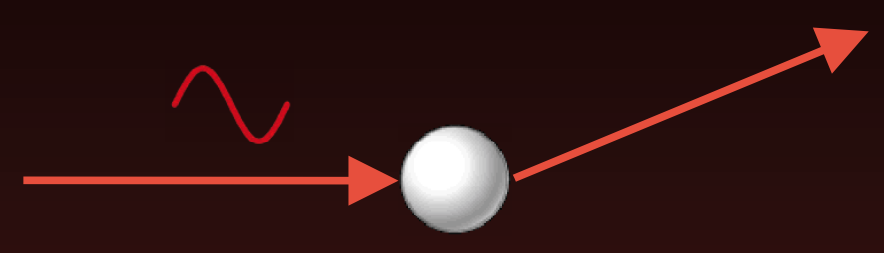

phase is maintained

multiple scattering

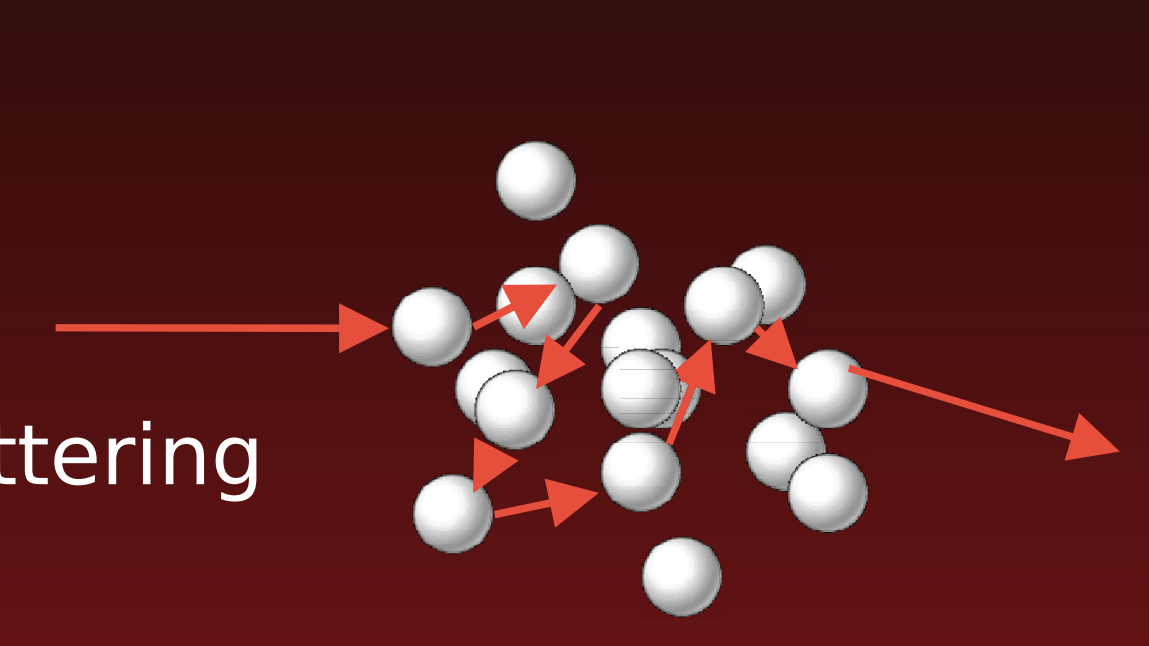

interference 


\section{Interference effects}

Speckle pattern
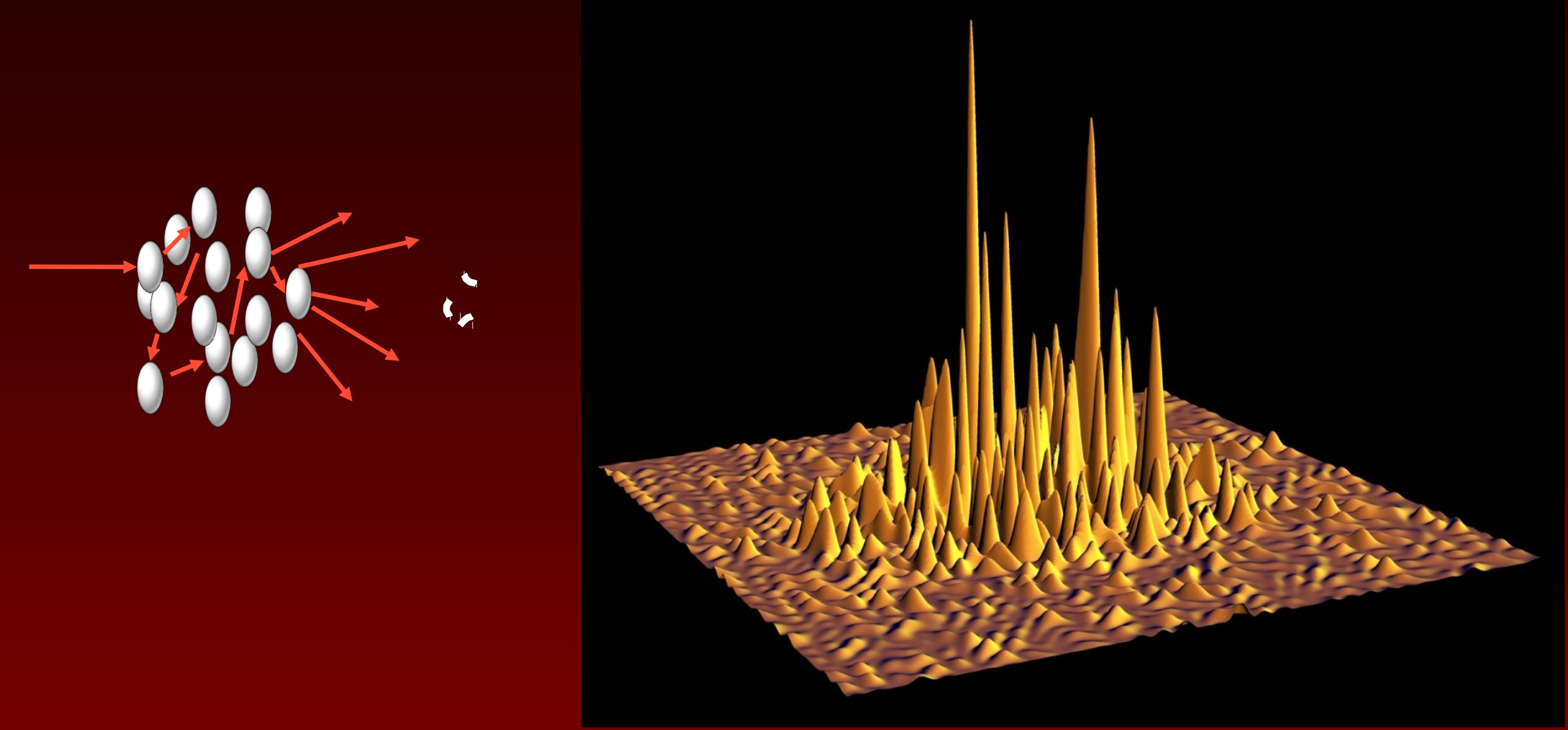


\section{Analogies electrons - photons}

Schrödinger's equation $\left[-\frac{h^{2}}{2 \mathrm{~m}} \nabla^{2}+V(\vec{r})\right] \Psi(\vec{r})=E \Psi(\vec{r})$

Maxwell's equations

$$
\left[-\nabla^{2}-\frac{\omega^{2} \varepsilon(\vec{r})}{c^{2}}\right] \vec{E}(\vec{r})=\frac{\omega^{2} \varepsilon}{c^{2}} \vec{E}(\vec{r})
$$




\section{Anderson localization}

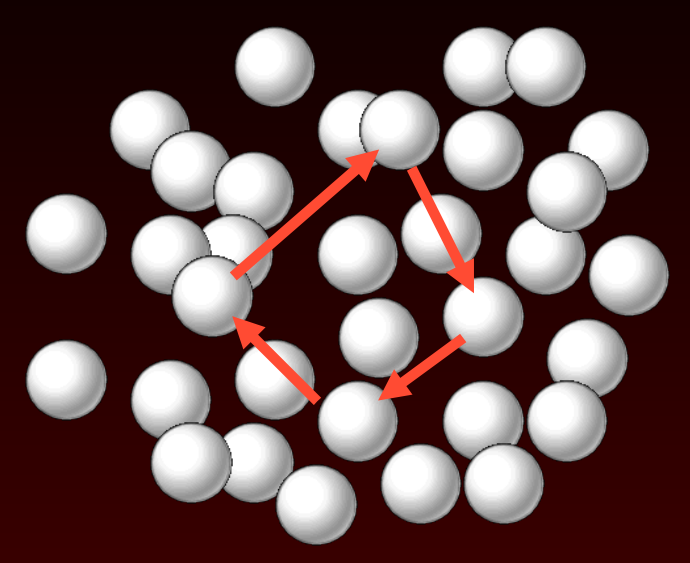

Very strongly scattering materials

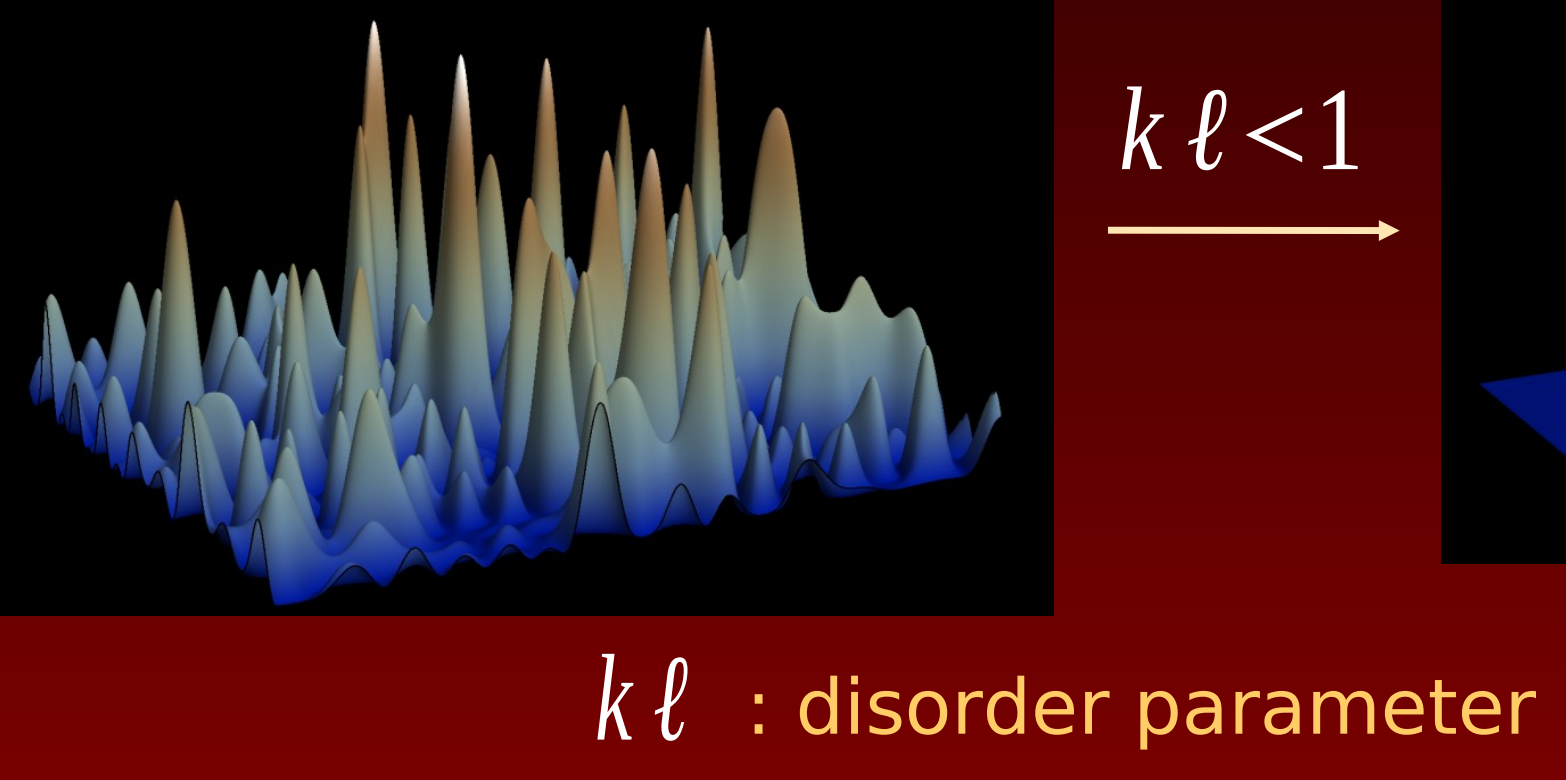

Nature 390, 671 (1997) Nature Physics 4, 359 (2008) 


\section{Localization in 1D}

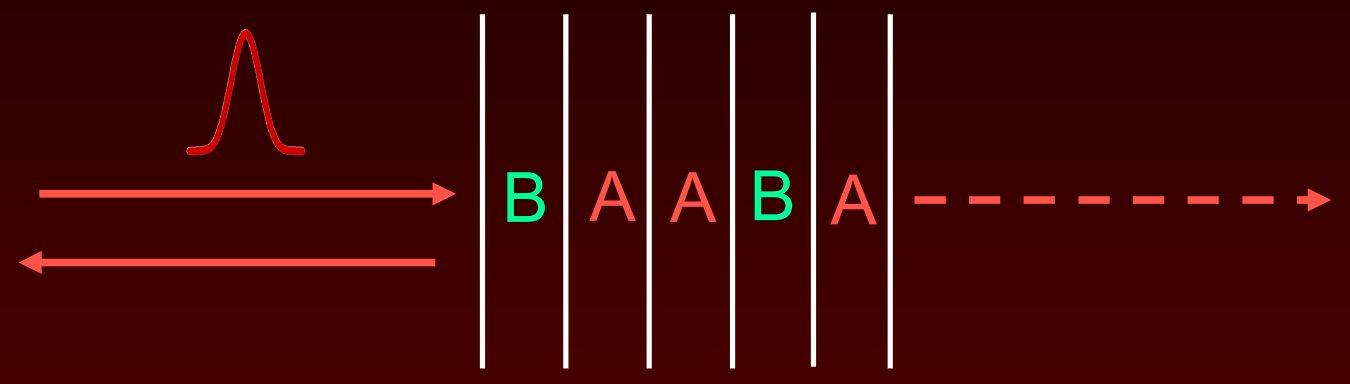

Random Stack of dielectric multilayers 


\section{Internal intensity distribution}

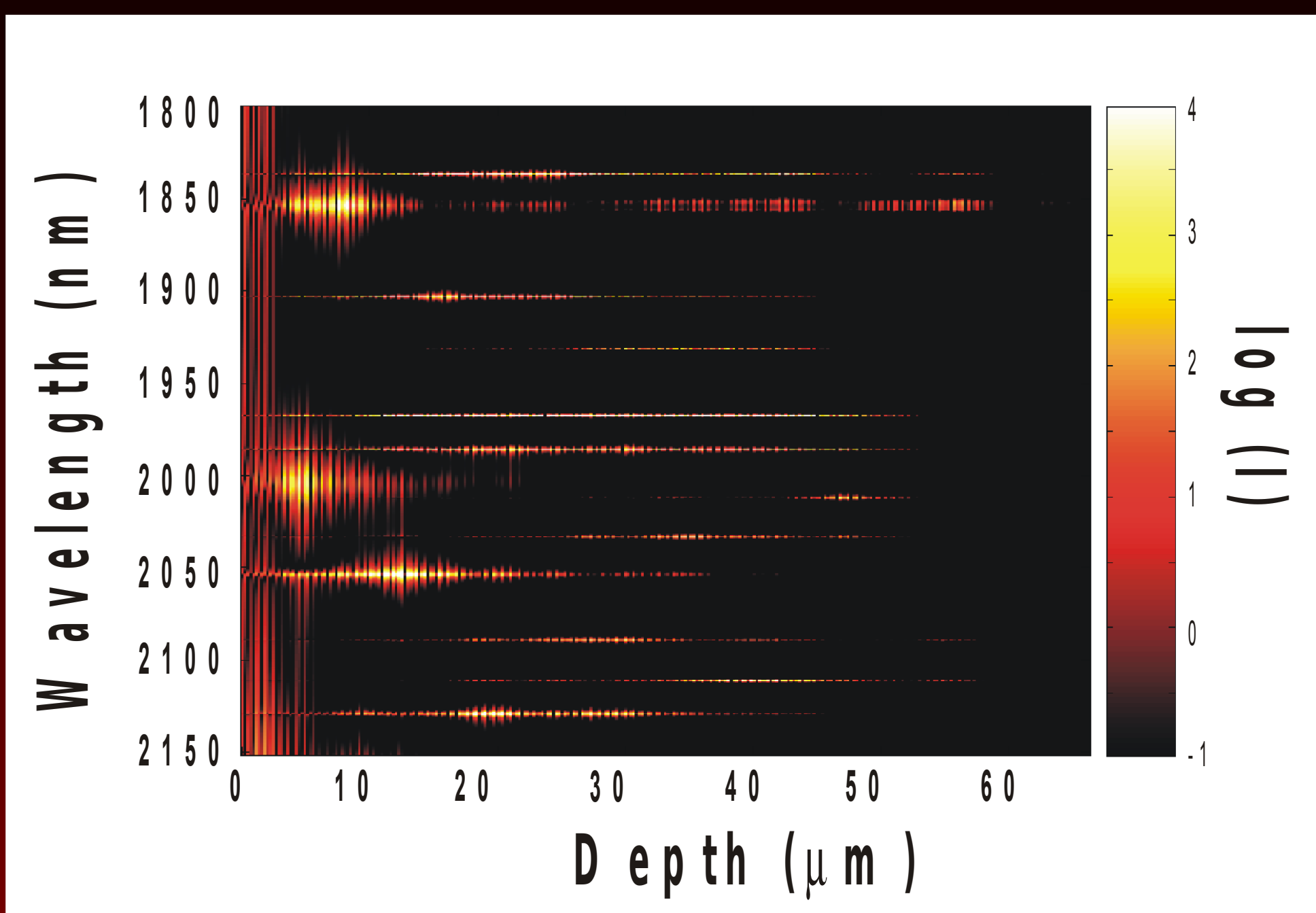




\section{Differences 1D vs 3D}

In 1D: always localization for any disordered system

(light waves, sound, Schrödinger waves)

In 3D: phase transition in disorder parameter

$k$ : wavevector, $\ell:$ mean free path 


\section{Lévy flights}




\section{Processes with Lévy statistics}

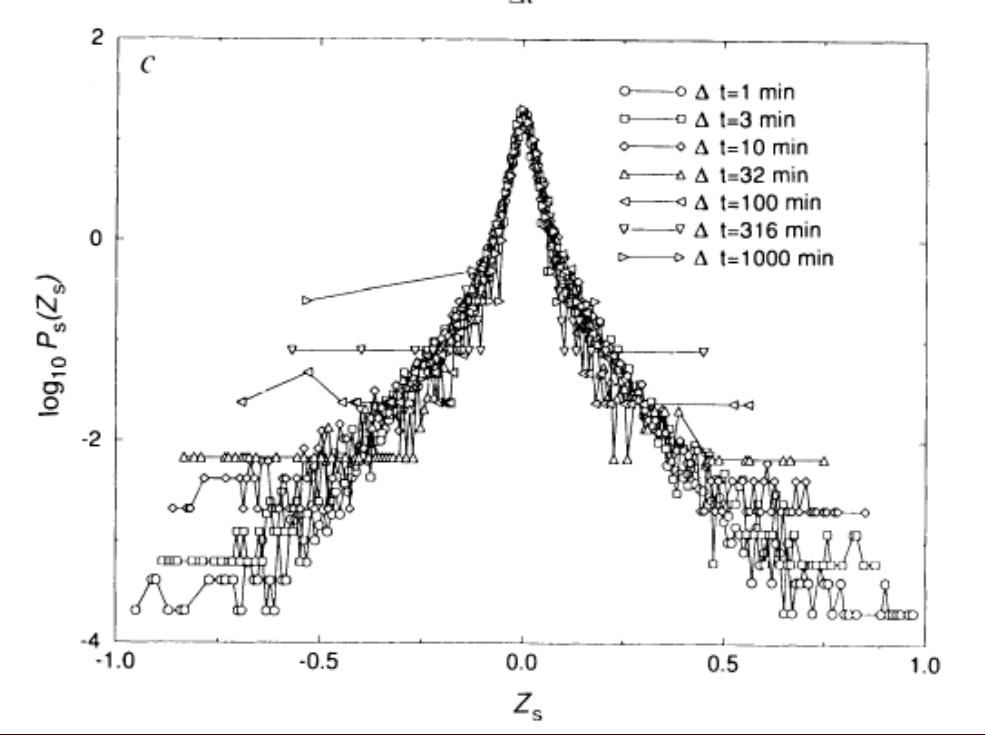

Stock market fluctuations

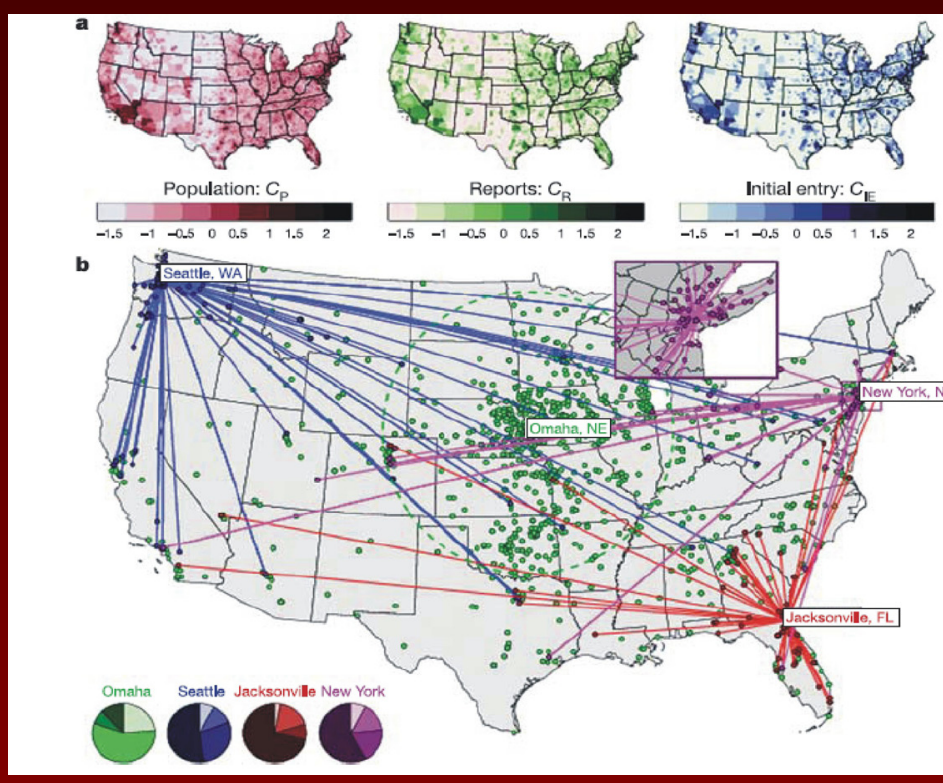

Human travel

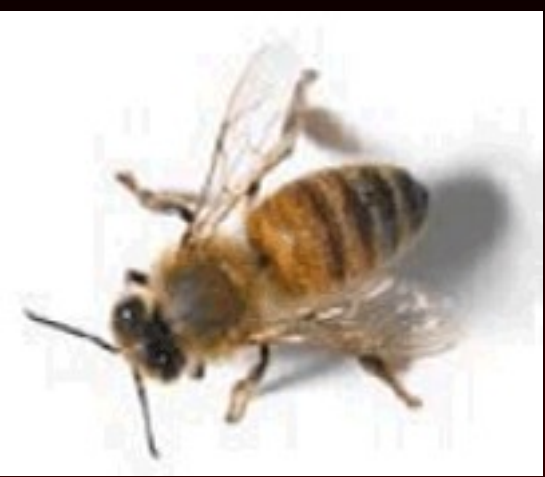

Animal foraging

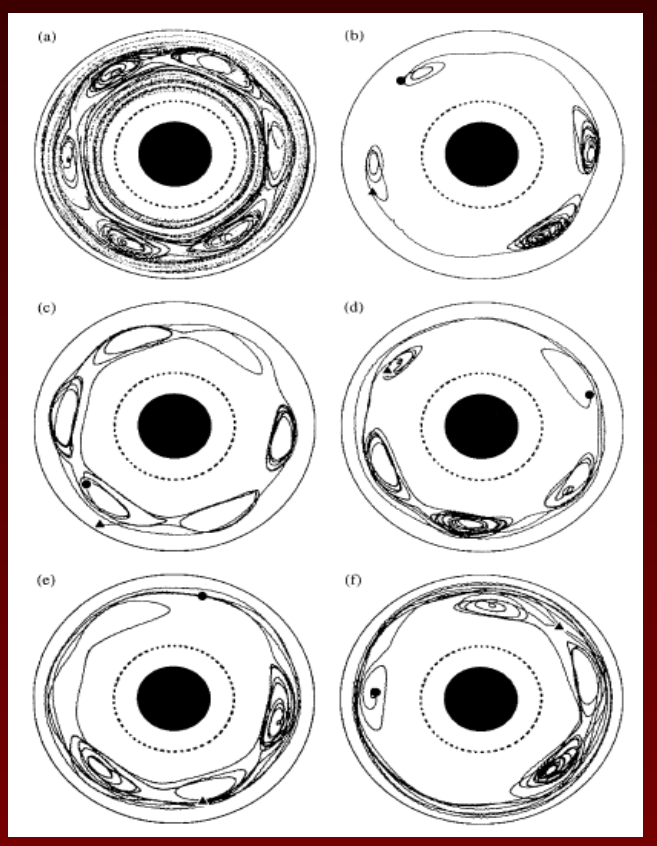

Turbulent flow 


\section{Stable distributions}

- Linear combination of elements remains in distribution

- Gaussian is limiting case with finite mean

- Rest has heavy tail asymptotic behaviour:

$$
P(z): \frac{1}{1+z^{\alpha+1}}
$$

$$
\alpha=1 \text { Cauchy distribution }
$$

- Infinite variance and (for $\alpha \leq 1$ ) also infinite mean 


\section{Gaussian random walk}

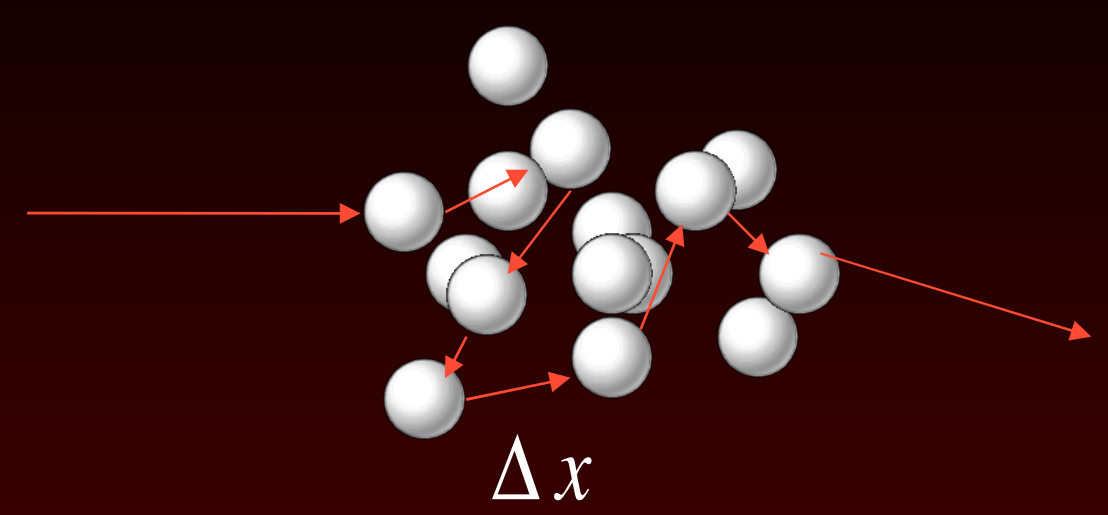

$\Delta x$ from distribution with finite average $\ell$ and variance

$$
\begin{gathered}
\text { Central limit theorem } \\
\sigma^{2} \propto D \cdot t \quad \text { Gaussian distribution }
\end{gathered}
$$

Diffusion process: $\left\langle x^{2}\right\rangle=D \cdot t \quad$ with $D=\frac{1}{3} v \ell$ 


\section{Lévy walk}

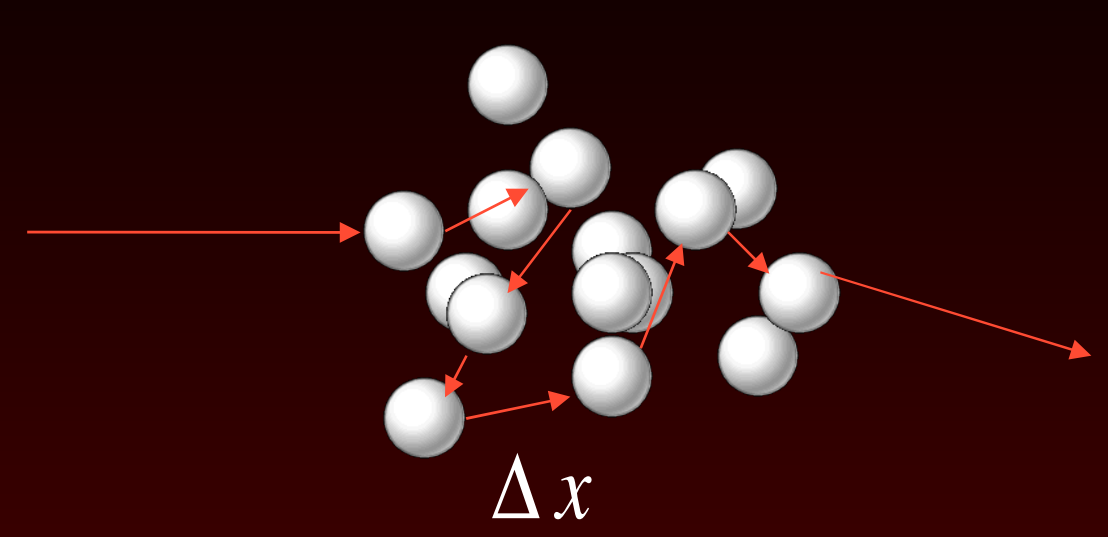

$\Delta x$ from stable distribution

Generalized

Generalized central limit theorem

diffusion process: $\left\langle x^{2}\right\rangle=D \cdot t^{\gamma} \quad$ with: $\gamma=3-\alpha$ for $1 \leq \alpha<2$

$$
\gamma=1: \text { Normal diffusion } \quad \gamma>1 \text { : Super diffusion }
$$




\section{Lévy walks for light waves}




\section{Lévy walk for light}

- Control step size $\langle z\rangle=\frac{1}{\sigma \cdot n} \quad \begin{aligned} & \sigma: \text { cross section } \\ & n \text { : density }\end{aligned}$

- Fractal particle size distibution?

$\longrightarrow$ does not work due to Rayleigh (Mie) scattering

- Engineer local particle density! 


\section{Lévy glass}

TiO2 nanoparticles + Glass Spheres + Sodium Silicate (liquid glass)

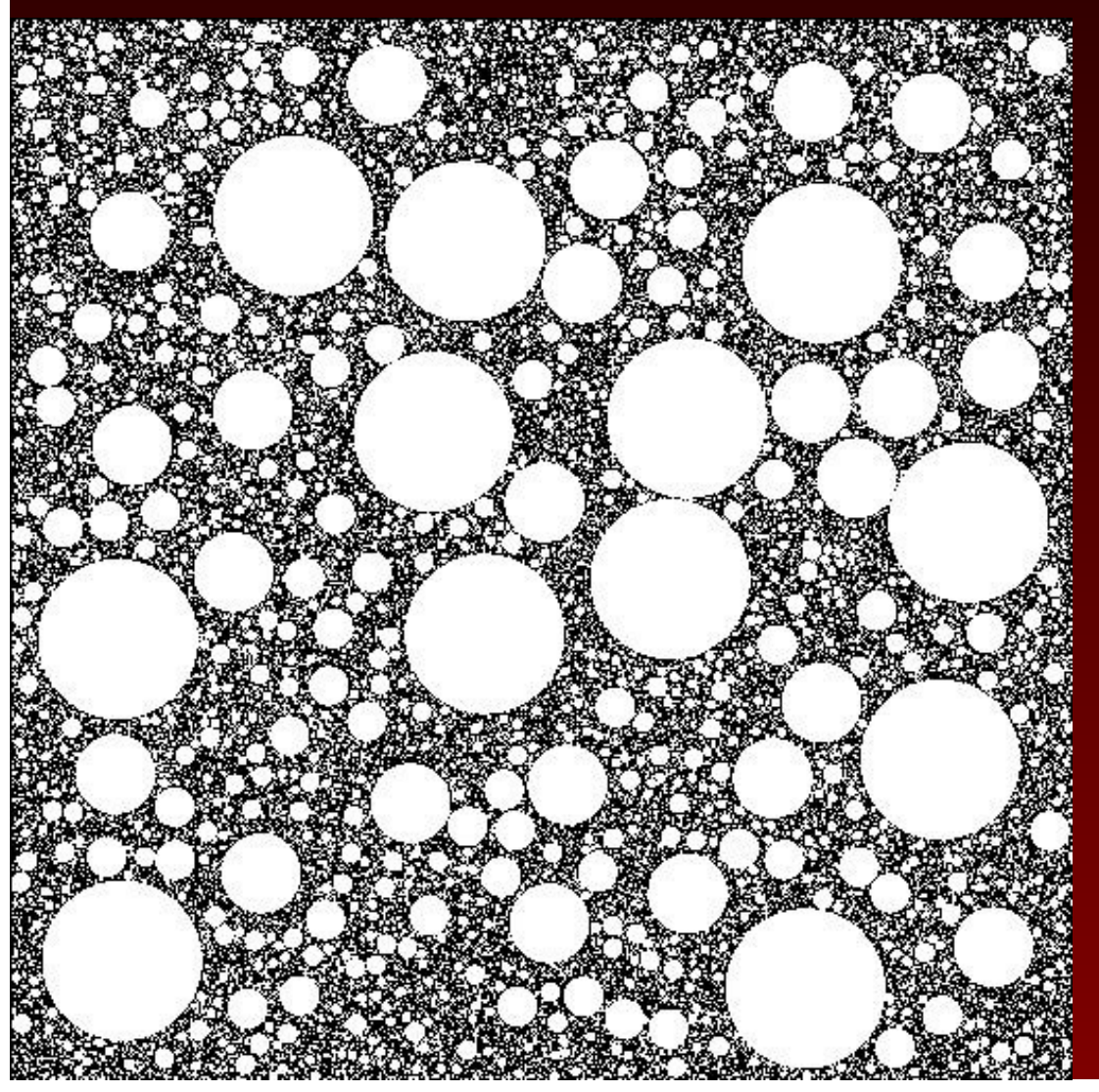

Glass and glass: index matched

Glass Spheres: introduce the density fluctuations

Diameter distribution:

$$
P(d) \sim \frac{1}{d^{2+\alpha}}
$$

( $d$ between 5 and $650 \mu \mathrm{m}$ ) 


\section{Lévy glass}

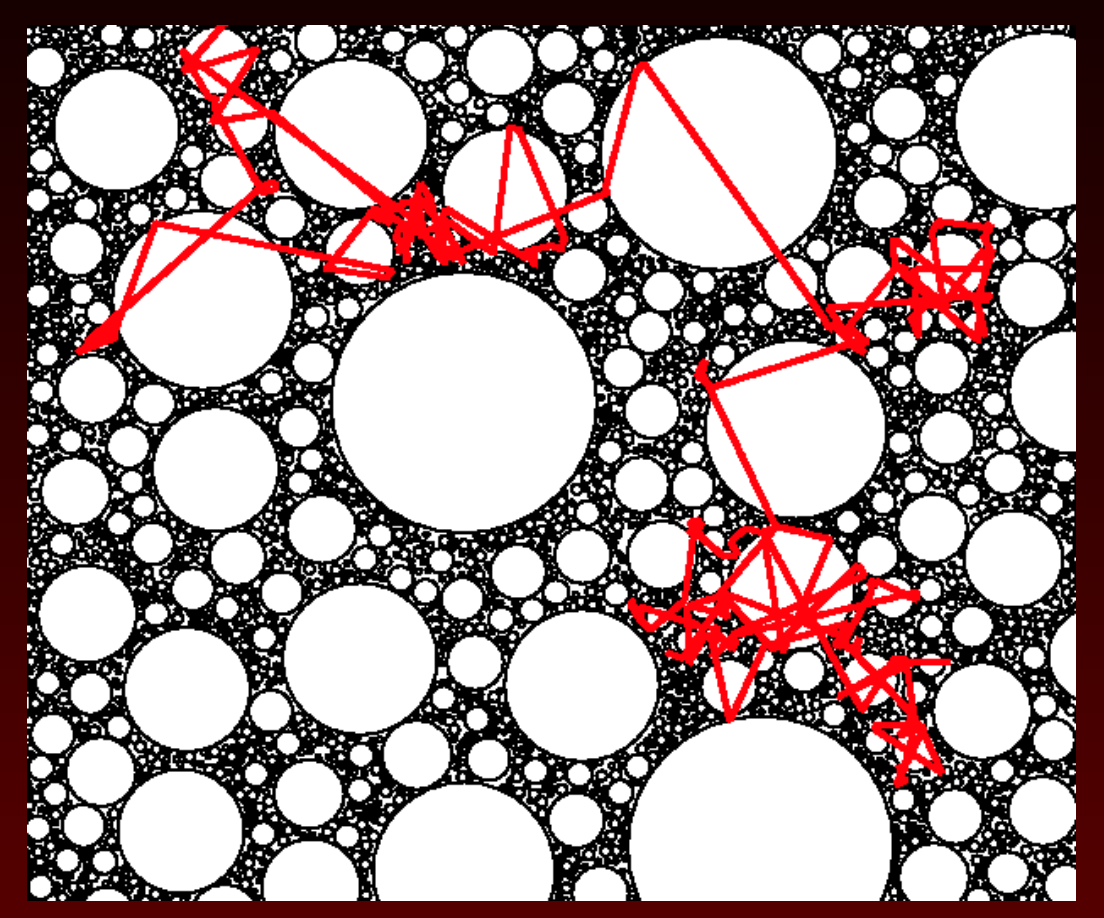

Lévy flight from multiple scattering on $\mathrm{TiO} 2$ particles

\section{nature}

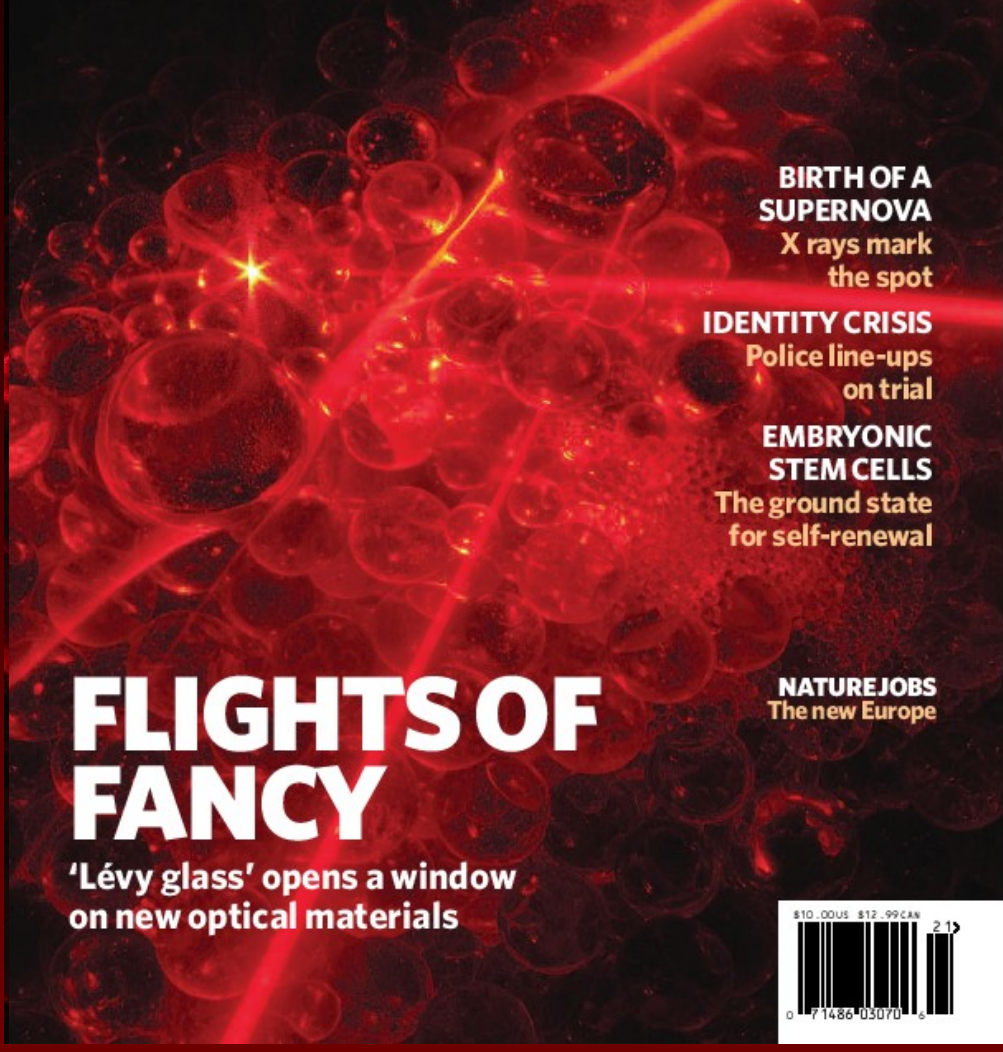

Nature 453, 498 (May 22, 2008) 


\section{Sample design}

Diameter distribution voids: $P(d) \sim \frac{1}{d^{2+\alpha}}$

Step length distribution:

$$
P(z) \sim \frac{1}{1+z^{\alpha+1}}
$$

Super diffusion: $\left\langle x^{2}\right\rangle=D \cdot t^{\gamma} \quad$ with: $\gamma=3-\alpha$ for $1 \leq \alpha<2$ 
Monte Carlo simulations 


\section{Monte Carlo simulations}

Free space Lévy flight with step length:

$$
P(z) \sim \frac{1}{1+z^{\alpha+1}}
$$

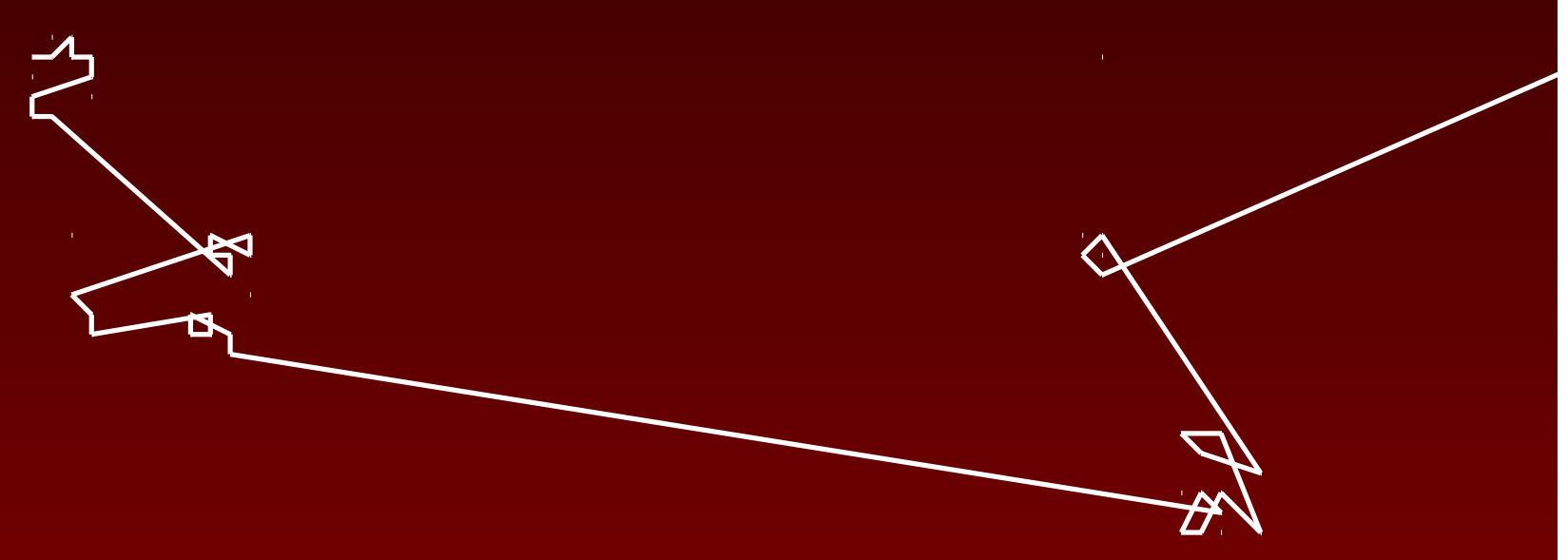




\section{Monte Carlo simulations}

Fixed geometry (quenched disorder)

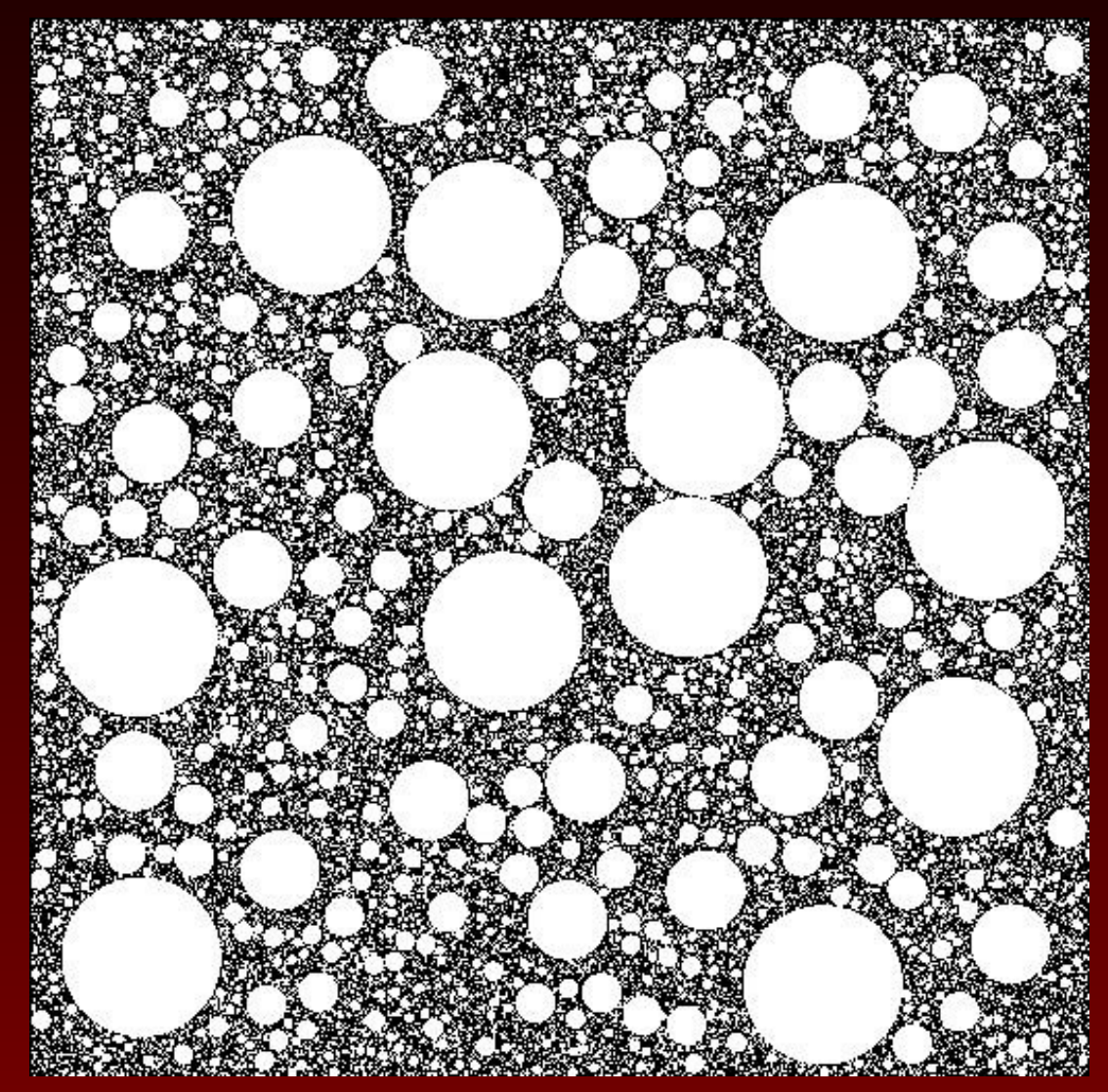


Placing spheres in a space

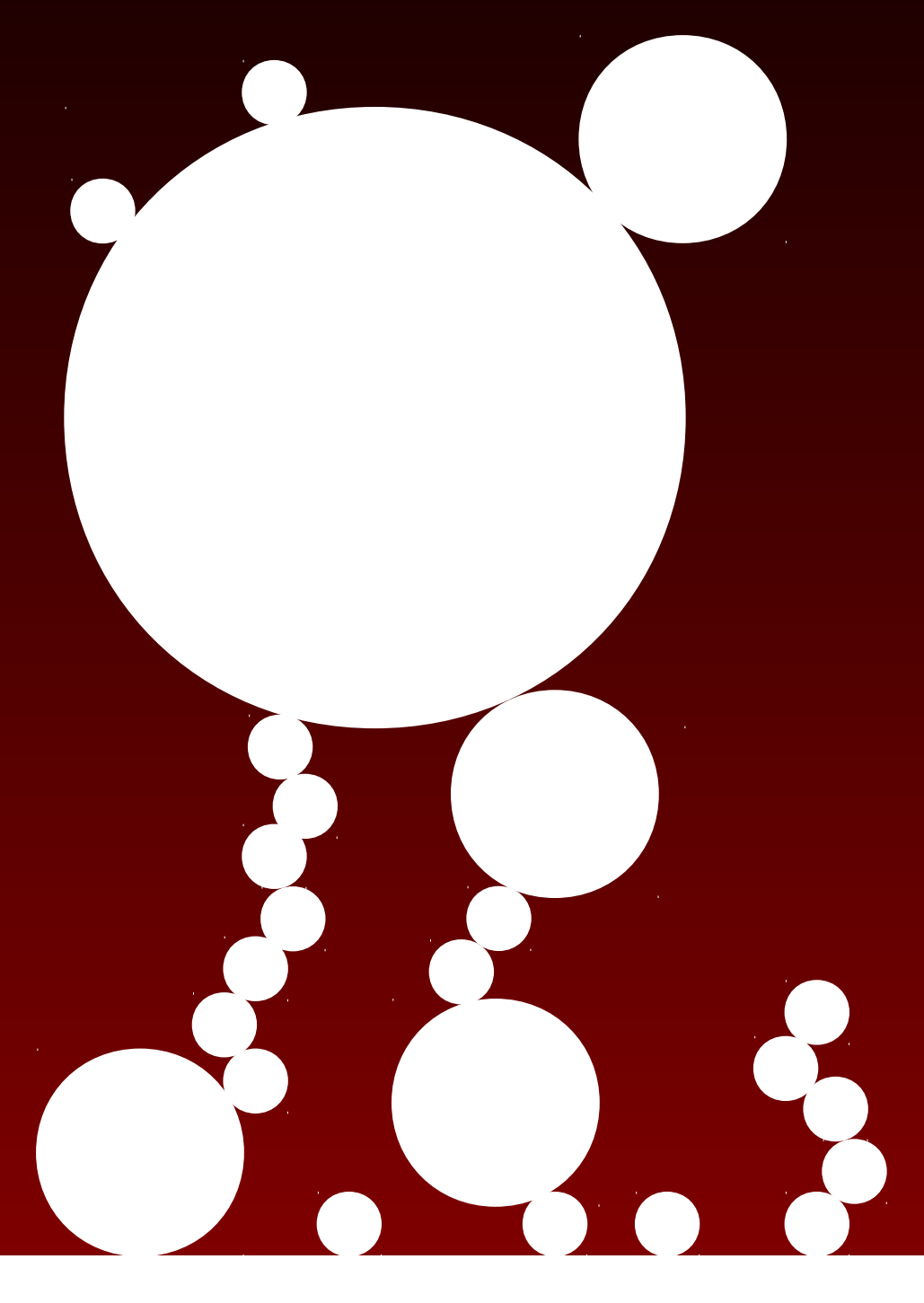




\section{Placing spheres in a space}

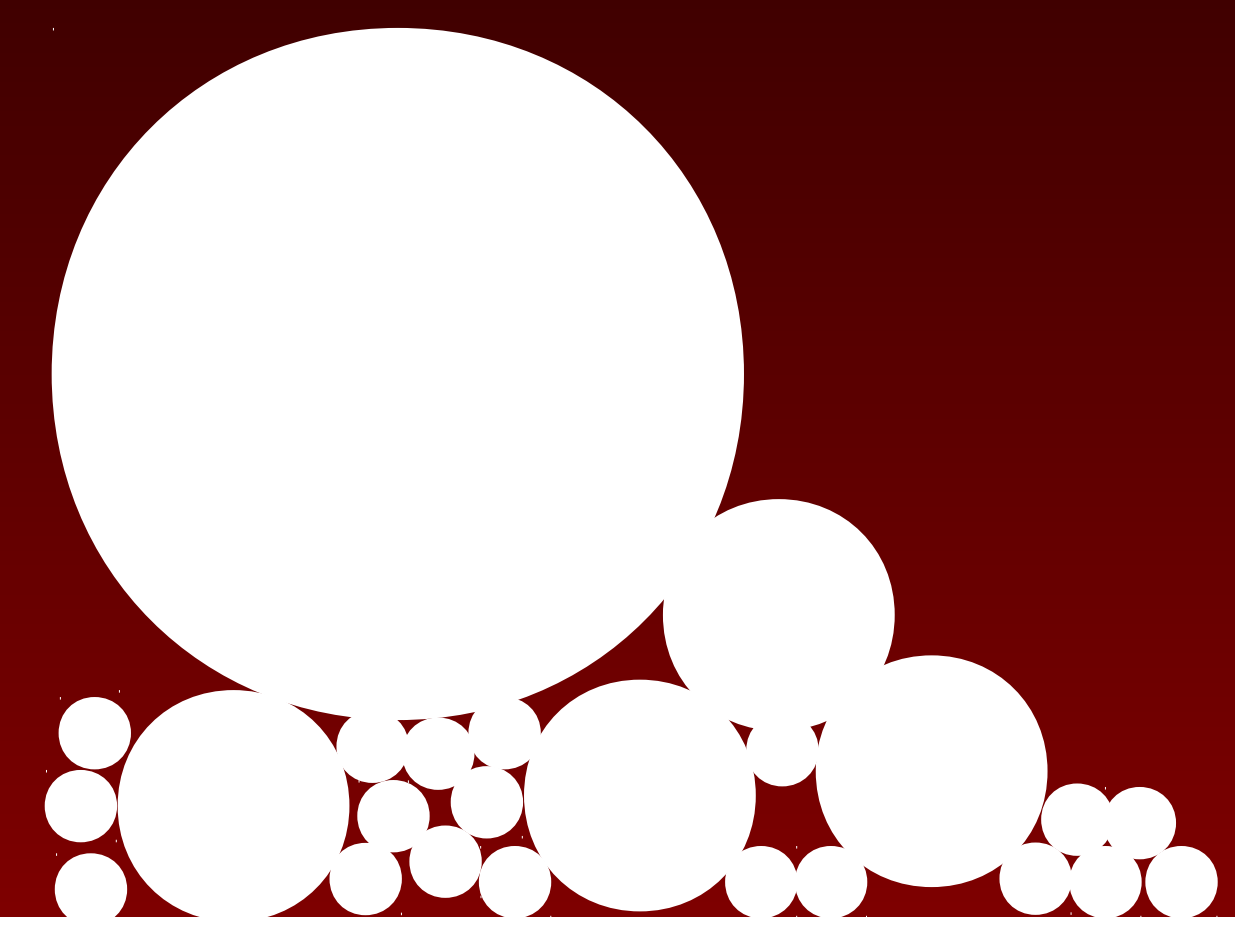




\section{Monte Carlo simulations}

Random walk with quenched disorder

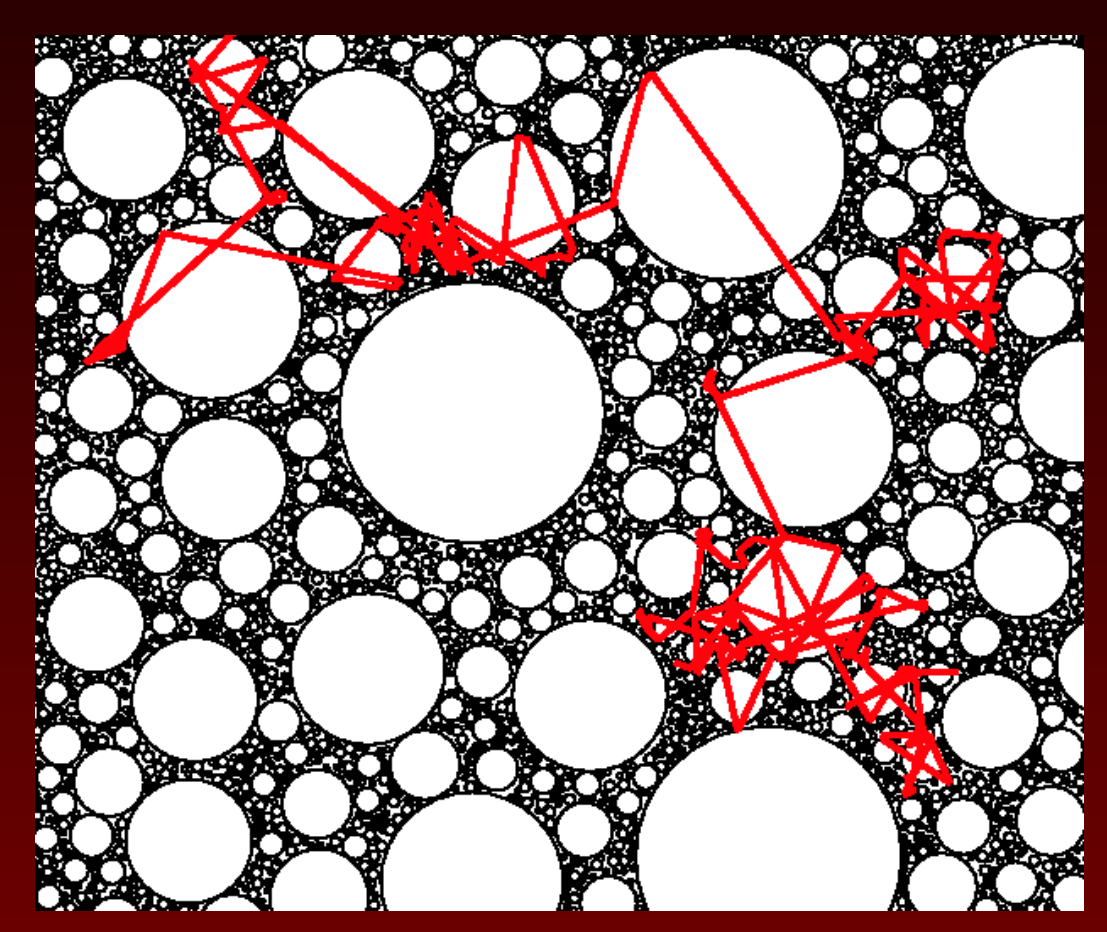




\section{Quenching effects}

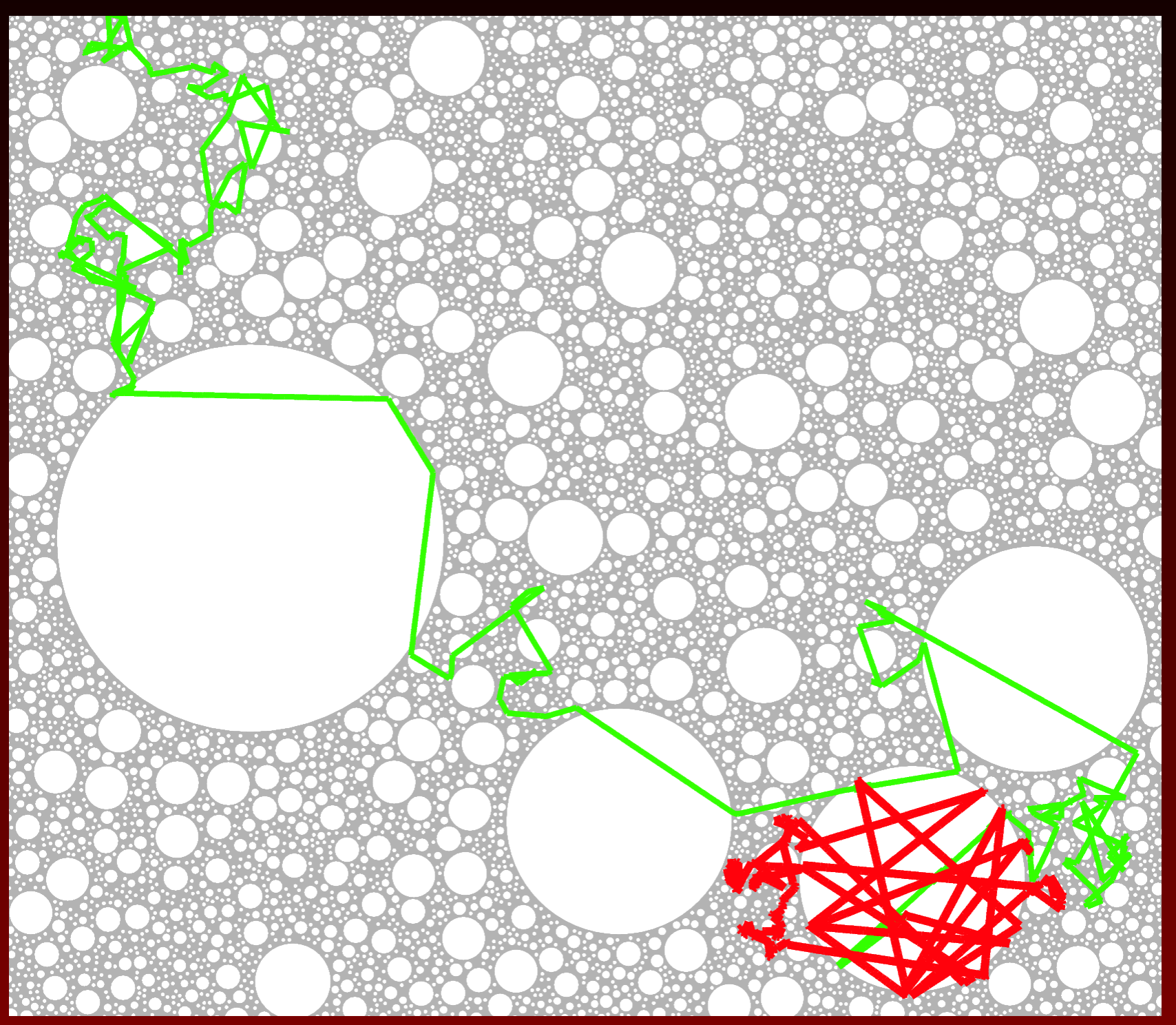




\section{Quenched vs annealed disorder}

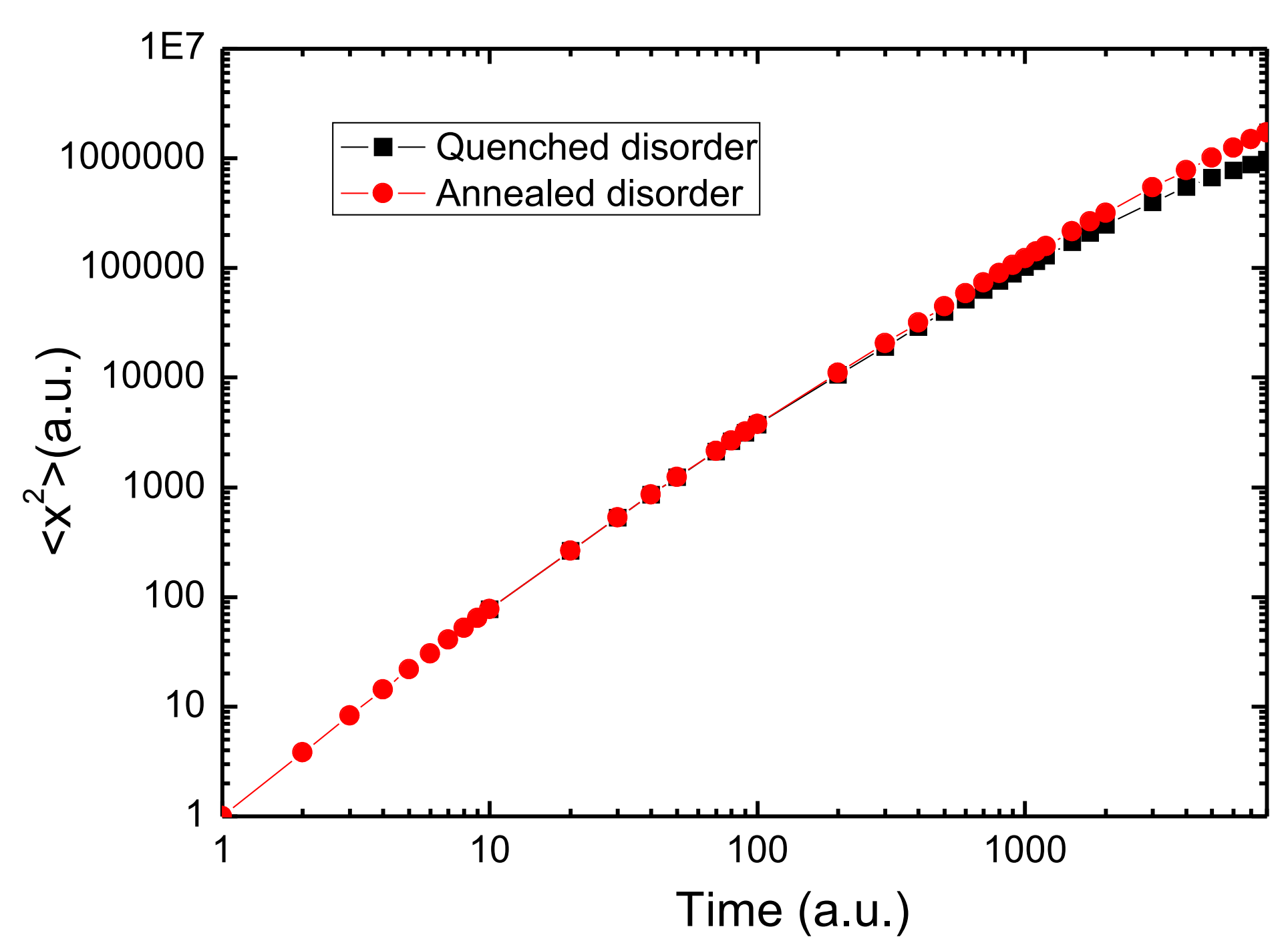


Experimental observations 


\section{Lévy glass}

TiO2 nanoparticles + Glass Spheres + Sodium Silicate (liquid glass)

TiO2 nanoparticles + Glass Spheres + Polymer matrix (n matched)

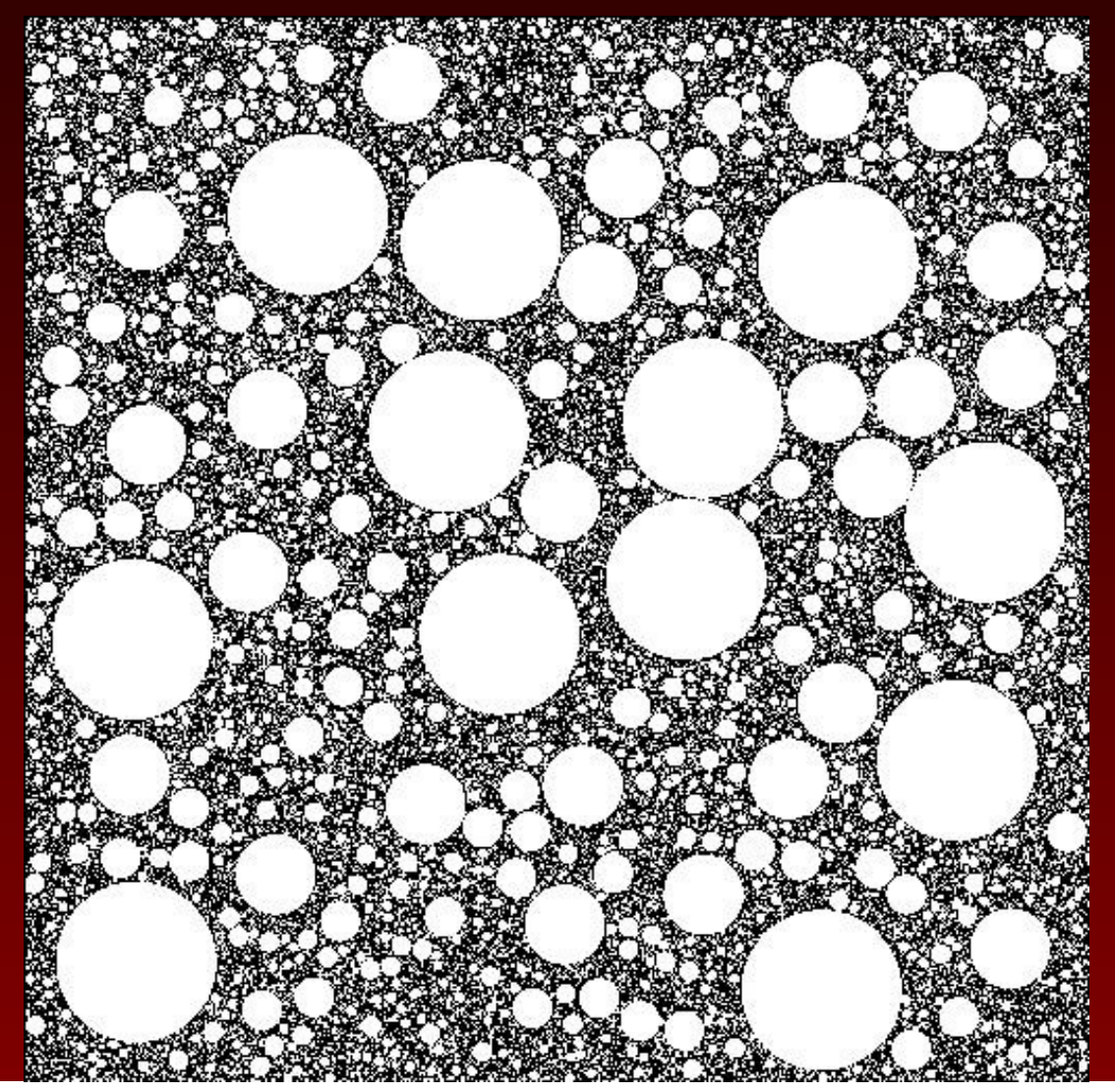




\section{Super diffusion}

Enhanced diffusive transmission

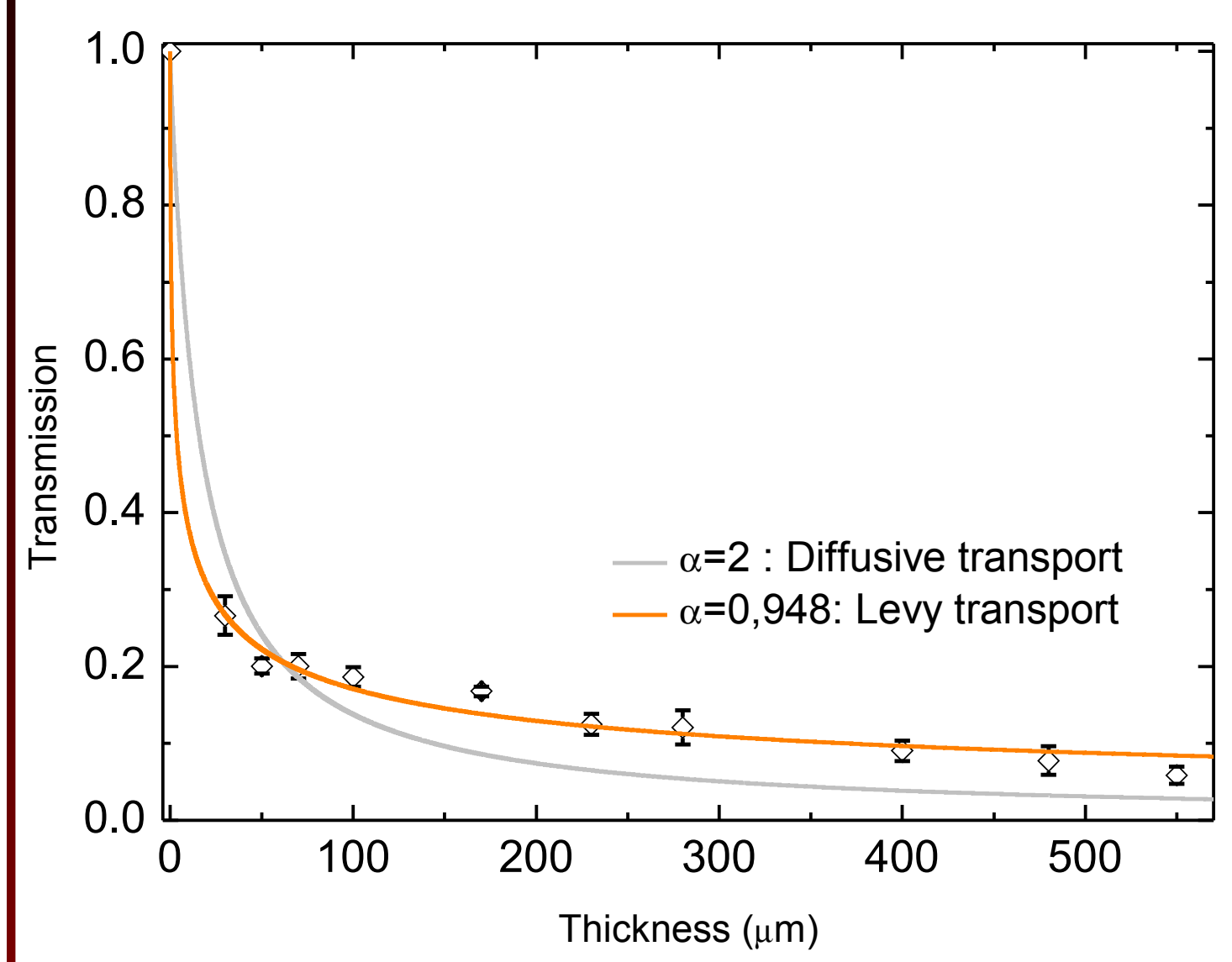

Generalised Ohm's law:

$$
T=\frac{1}{1+a L^{\alpha / 2}}
$$




\section{Transmission profiles}

Diffusive case: small fluctuations
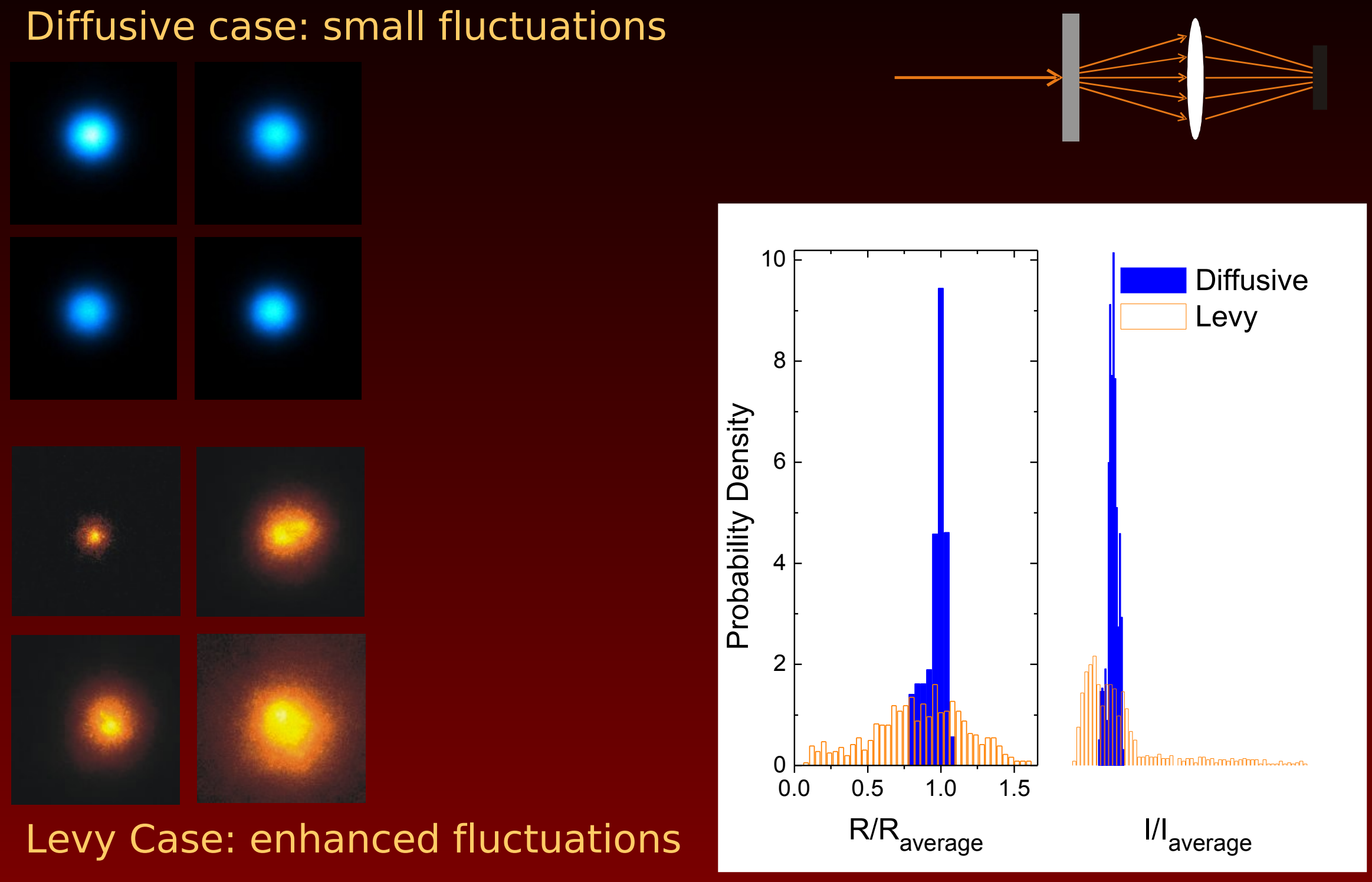


\section{Enhanced spreading}

Diffusive Case: Almost-gaussian profile Lévy Case: Cusped spatial profile
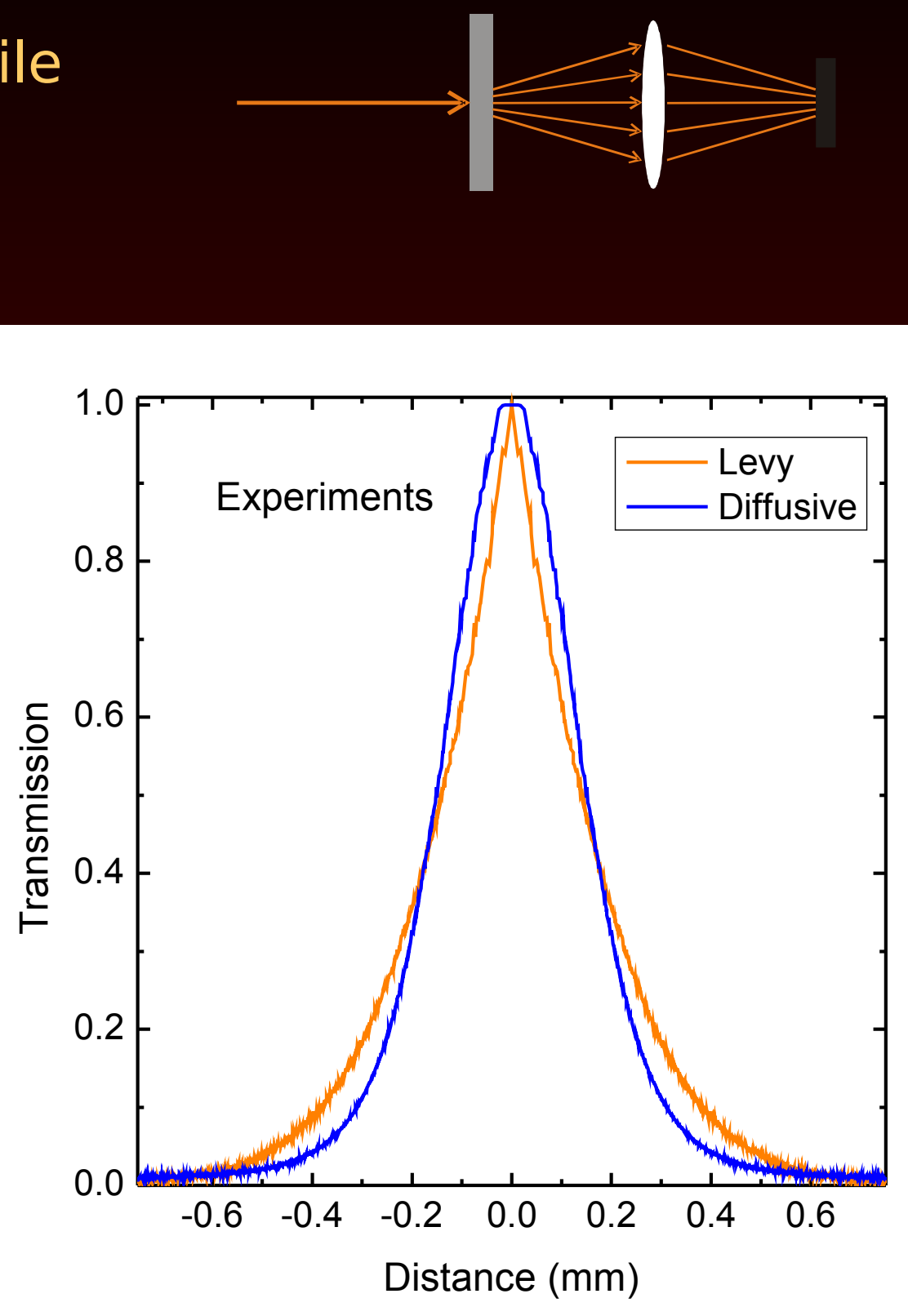


\section{Summary}

- Transport of light in random systems

- How to realize non-gaussian optical disorder: Levy glass

- Superdiffusion of light, possibility to study optical Levy flights

- Open questions:

- Unknown properties of Lévy flights (e.g. finite-size effects)

- Weak localization (coherent backscattering), strong localization, speckle correlations, etc.. 


\section{Micro and nano photonics group}

European Lab. For Non-linear Spectroscopy (LENS)

INO-CNR, Unív. of Florence

www.compléxphotonics.org

Pierre Barthelemy

Matteo Burresi

Francesca Intonti

Rajesh Kumar

lacopo Malfanti

Vitantonio Matarazzo
Camilla Parmeggiani Francesco Riboli Romolo Savo Radha Vivekananthan Kevin Vynck

Jacopo Berlototti Silvia Vignolini
Stefano Cavalieri Marcello Colocci Massimo Gurioli Stefano Lepri Roberto Livi Roberto Righini 